\title{
Logic-based switching control of a nonholonomic system with parametric modeling uncertainty *
}

\author{
João P. Hespanha ${ }^{\dagger} \quad$ Daniel Liberzon $\ddagger \quad$ A. Stephen Morse ${ }^{\ddagger}$ \\ ${ }^{\dagger}$ Dept. of Electrical Eng. and Computer Science \\ University of California \\ Berkeley, CA 94720-1770 \\ hespanha@eecs. berkeley.edu \\ ${ }^{\ddagger}$ Dept. of Electrical Engineering \\ Yale University \\ New Haven, CT 06520-8267 \\ \{liberzon, morse\}@sysc.eng.yale.edu
}

\begin{abstract}
This paper is concerned with control of nonholonomic systems in the presence of parametric modeling uncertainty. The specific problem considered is that of parking a wheeled mobile robot of unicycle type with unknown parameters, whose kinematics can be described by Brockett's nonholonomic integrator after an appropriate state and control coordinate transformation. We employ the techniques of supervisory control to design a hybrid feedback control law that solves this problem.
\end{abstract}

Keywords: Nonholonomic system; modeling uncertainty; hybrid feedback control; estimator-based supervisor.

\section{Introduction}

Control systems with nonholonomic motion constraints have been extensively studied in the recent years, particularly in the context of robotics. Robotic manipulators, especially mobile ones, are described by complicated models about which there is likely to be significant uncertainty [4]. This is just one source of motivation for studying the challenging problem of controlling nonholonomic mechanical systems in the presence of modeling uncertainty. The status of this problem as of December 1995 is perhaps best expressed by the following quote from the survey article by Kolmanovsky and McClamroch [14]: "There are many important research problems for nonholonomic control systems that have been little studied. Here we identify the problem of control of nonholonomic systems when there are model uncertainties, as arise from parameter variations or from neglected dynamics. It is not necessary to motivate the importance of this problem, but it is curious that there is little published literature that deals directly with these questions". In the last few years this problem has received considerable attention in the literature, and several adaptive control strategies for uncertain nonholonomic systems have been proposed [3, 4, 12, 13, 20, 21].

The goal of this paper is to demonstrate that a promising alternative to conventional adaptive control for nonholonomic systems is provided by the techniques of supervisory control (see [9] for the most recent

*Submitted to Systems and Control Letters (special issue on hybrid systems). This research was supported by ARO DAAH0495-1-0114, NSF ECS 9634146, and AFOSR F49620-97-1-0108. 
exposition and references). The basic idea behind this approach is to employ logic-based switching among a family of candidate control laws. There is considerable flexibility in the choice of these control laws, which leads us to believe that various control strategies which have been developed for nonholonomic systems can be put to use in the case when modeling uncertainty is present. The results reported in $[10,18]$ on designing hybrid control laws for nonholonomic systems (which provide one way of dealing with the problem that these systems fail to satisfy Brockett's necessary condition for smooth feedback stabilizability [2]) are of particular relevance. This line of research fits together nicely with the framework proposed here because, as illustrated below, in the supervisory control setting it is possible to switch in a hybrid fashion between candidate control laws that are themselves hybrid.

In what follows, we consider a prototype example of an uncertain nonholonomic control system, whose kinematics can be described by Brockett's nonholonomic integrator after an appropriate state and control coordinate transformation. We demonstrate how the supervisory control approach can be used to design a hybrid feedback control law that drives the state of the system to zero. The control algorithm employs a version of the so-called hysteresis switching logic, and in this sense is reminiscent of the hybrid control law used in [10] to stabilize the nonholonomic integrator with no modeling uncertainty. It follows from the work of Murray and Sastry [17] that any kinematic completely nonholonomic system with three states and two control inputs can be converted to the nonholonomic integrator by means of a state and control transformation. This result suggests that a fairly general class of nonholonomic systems can be treated by the method proposed in this paper.

The problem to be addressed is formulated in Section 2. In Section 3 we construct a hybrid control law based on estimator-based supervision and give a proof of convergence for the case when the unknown parameters take values in a finite set. In Section 4 we treat the somewhat simpler problem of practical regulation. The corresponding problems for the case of an infinite parameter set are discussed in Section 5. We make some concluding remarks in Section 6. The paper is self-contained; no previous knowledge of supervisory control methods is necessary for its understanding. In the Appendix, to make the paper more accessible, we consider a simple example of a (linear) supervisory control system to give an informal overview of the ideas involved.

\section{Problem formulation}

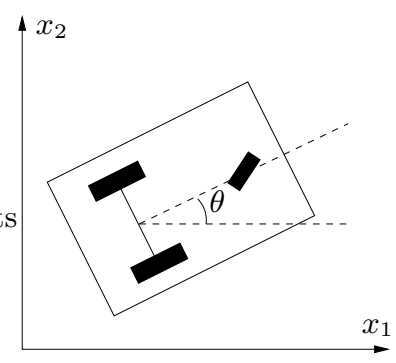

Figure 1: A wheeled mobile robot

The problem we consider is that of parking a wheeled mobile robot of unicycle type (see, e.g., [14]). Let $x_{1}, x_{2}$ be the coordinates of the point in the middle of the rear axle, and let $\theta$ denote the angle that the vehicle makes with the $x_{1}$-axis (see Figure 1 ). The front wheel turns freely and balances the front end of the 
robot above the ground. When the same angular velocity is applied to both rear wheels, the robot moves straight forward. When the angular velocities applied to the rear wheels are distinct, the robot turns. The kinematics of the robot can be modeled by the equations

$$
\begin{aligned}
\dot{x}_{1} & =p_{1}^{*} w_{1} \cos \theta \\
\dot{x}_{2} & =p_{1}^{*} w_{1} \sin \theta \\
\dot{\theta} & =p_{2}^{*} w_{2}
\end{aligned}
$$

where $p_{1}^{*}$ and $p_{2}^{*}$ are positive parameters determined by the radius of the rear wheels and the distance between them, and $w_{1}$ and $w_{2}$ are the control inputs. The case we are interested in is when the actual values of $p_{1}^{*}$ and $p_{2}^{*}$ are not precisely known. In the sequel we assume that the pair $p^{*}:=\left(p_{1}^{*}, p_{2}^{*}\right)$ belongs to a set $\mathcal{P}:=\mathcal{P}_{1} \times \mathcal{P}_{2}$, where $\mathcal{P}_{1}$ and $\mathcal{P}_{2}$ are finite (or infinite but compact) subsets of $(0, \infty)$. We will denote the state $\left(x_{1}, x_{2}, \theta\right)^{T}$ of the unicycle by $\mathbf{x}_{\mathrm{u}}$ and the input vector $\left(w_{1}, w_{2}\right)^{T}$ by $\mathbf{w}$. The problem of parking the vehicle amounts to making $x_{1}, x_{2}$, and $\theta$ tend to zero by means of applying a state feedback (the whole state $\mathbf{x}_{\mathrm{u}}$ being available for control).

Consider the state coordinate transformation

$$
\begin{aligned}
& x=\left(x_{1} \cos \theta+x_{2} \sin \theta\right) \\
& y=\theta \\
& z=2\left(x_{1} \sin \theta-x_{2} \cos \theta\right)-\theta\left(x_{1} \cos \theta+x_{2} \sin \theta\right)
\end{aligned}
$$

It is easy to verify that (2) defines a global diffeomorphism that preserves the origin. Consider also the family of control transformations given, for each $p \in \mathcal{P}$, by

$$
\begin{aligned}
& u_{p}=f_{p}\left(\mathbf{x}_{\mathrm{u}}, \mathbf{w}\right):=p_{1} w_{1}-p_{2} w_{2}\left(x_{1} \sin \theta-x_{2} \cos \theta\right) \\
& v_{p}=g_{p}\left(\mathbf{x}_{\mathbf{u}}, \mathbf{w}\right):=p_{2} w_{2}
\end{aligned}
$$

For $p=p^{*}$ the transformed equations are those of Brockett's nonholonomic integrator [2]

$$
\begin{aligned}
& \dot{x}=u_{p^{*}} \\
& \dot{y}=v_{p^{*}} \\
& \dot{z}=x v_{p^{*}}-y u_{p^{*}}
\end{aligned}
$$

We will denote $(x, y, z)^{T}$ by $\mathbf{x}_{\mathbf{i}}$. Following [5] (see also [18]), we further transform the state and control variables according to

$$
\begin{aligned}
& x=r \cos \psi \\
& y=r \sin \psi
\end{aligned} \quad\left(\begin{array}{l}
u_{p} \\
v_{p}
\end{array}\right)=\left(\begin{array}{cc}
\cos \psi & -\sin \psi \\
\sin \psi & \cos \psi
\end{array}\right)\left(\begin{array}{l}
\bar{u}_{p} \\
\bar{v}_{p}
\end{array}\right)
$$

to obtain the following equations in the new cylindrical coordinates:

$$
\begin{aligned}
& \dot{r}=\bar{u}_{p^{*}} \\
& \dot{\psi}=\bar{v}_{p^{*}} / r \\
& \dot{z}=r \bar{v}_{p^{*}}
\end{aligned}
$$

(of course, the above transformation is only defined when $x^{2}+y^{2} \neq 0$ ). If the value of $p^{*}$ were known, one could apply the controls

$$
\bar{u}_{p^{*}}=-r^{2}, \quad \bar{v}_{p^{*}}=-z
$$


which result in the system

$$
\begin{aligned}
\dot{r} & =-r^{2} \\
\dot{z} & =-z r \\
\dot{\psi} & =-\frac{z}{r}
\end{aligned}
$$

Thus if $r(0) \neq 0$, it is not hard to see that $r(t), z(t) \rightarrow 0$. If $r(0)=0$, we could apply some control law that moves the state $\mathbf{x}_{\mathrm{i}}$ of (4) away from the $z$-axis (e.g., $u_{p^{*}}=v_{p^{*}}=1$ ) for a certain amount of time, and then switch to the control defined by (6). This would result in a simple hybrid control law that drives $\mathbf{x}_{\mathbf{i}}$, and consequently $\mathbf{x}_{\mathrm{u}}$, to zero. In fact, it is even possible to achieve asymptotic stability in the Lyapunov sense. Although the control law (6) is not the best one available (for example, it does not achieve exponential convergence), it provides motivation for the subsequent developments.

Since the actual parameter values $p_{1}^{*}$ and $p_{2}^{*}$ are unknown, the above control strategy cannot be implemented. Instead, we will develop a hybrid feedback law of the form $\mathbf{w}=\boldsymbol{\rho}_{\sigma}$, where $\left\{\boldsymbol{\rho}_{p}: p \in \mathcal{P}\right\}$ is a family of candidate control laws, each designed for a specific value of $p \in \mathcal{P}$, and $\sigma:[0, \infty) \rightarrow \mathcal{P}$ is a piecewise constant switching signal that determines, at each instant of time, which one of these control laws should be used.

\section{Estimator-based supervisory control}

The purpose of this section is to develop a "high-level" controller called a supervisor which, without a priori knowledge of $p^{*}$, is capable of orchestrating the switching among a suitably defined family of candidate control laws so as to cause the state $\mathbf{x}_{\mathrm{u}}$ of the system (1) to tend to zero. In this section and the next one we assume that the set $\mathcal{P}$ is finite. The reader who is not familiar with the ideas of supervisory control is encouraged to consult the Appendix. The supervisor consists of four subsystems (see Figure 2):

multi-estimator - a dynamical system whose inputs are $\mathbf{w}$ and $\mathbf{x}_{\mathrm{i}}$ and whose outputs are $\mathbf{x}_{p}, p \in \mathcal{P}$, where each $\mathbf{x}_{p}$ is a suitably defined estimate of $\mathbf{x}_{\mathbf{i}}$ which would be asymptotically correct if $p$ were equal to $p^{*}$.

multi-controller - a dynamical system whose inputs are $\mathbf{x}_{p}$ and the estimation errors $\mathbf{e}_{p}:=\mathbf{x}_{p}-\mathbf{x}_{\mathbf{i}}, p \in \mathcal{P}$, and whose outputs are control signals $\mathbf{w}_{p}, p \in \mathcal{P}$ generated by the candidate control laws $\boldsymbol{\rho}_{p}, p \in \mathcal{P}$.

performance signal generator - a dynamical system whose inputs are the estimation errors $\mathbf{e}_{p}$ and whose outputs $\pi_{p}, p \in \mathcal{P}$ are suitably "normed" values of the estimation errors called performance signals.

switching logic - a dynamical system whose inputs are the performance signals $\pi_{p}$ and whose output is a switching signal $\sigma$ which is used to define the control law $\mathbf{w}=\boldsymbol{\rho}_{\sigma}$.

The underlying decision-making strategy used by the supervisor basically consists in selecting for $\sigma$, from time to time, the candidate control index $q$ whose corresponding performance signal $\pi_{q}$ is currently the smallest among the $\pi_{p}, p \in \mathcal{P}$. The motivation for this heuristic idea is as follows: the process model whose associated performance signal is the smallest "best" approximates what the process is, and thus the candidate control law designed on the basis of that model can be expected to do the best job of controlling the process. 


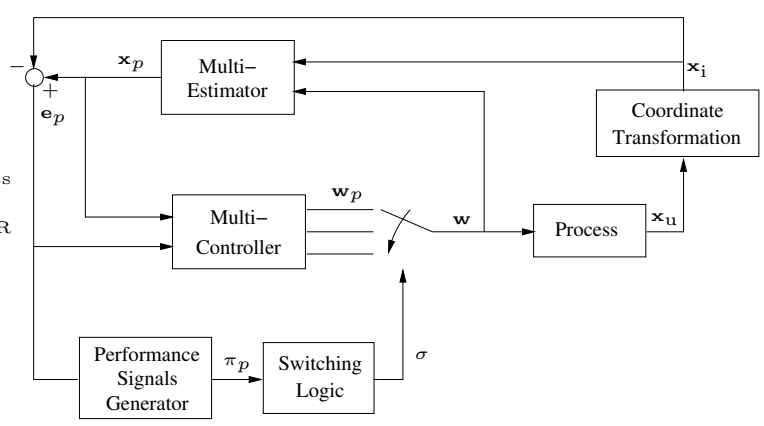

Figure 2: Supervisory control system

\subsection{Multi-Estimator}

In view of (4), we introduce the family of estimator equations

$$
\begin{aligned}
& \dot{x}_{p}=-\left(x_{p}-x\right)+f_{p}\left(\mathbf{x}_{\mathrm{u}}, \mathbf{w}\right) \\
& \dot{y}_{p}=-\left(y_{p}-y\right)+g_{p}\left(\mathbf{x}_{\mathrm{u}}, \mathbf{w}\right) \\
& \dot{z}_{p}=-\left(z_{p}-z\right)+x g_{p}\left(\mathbf{x}_{\mathrm{u}}, \mathbf{w}\right)-y f_{p}\left(\mathbf{x}_{\mathrm{u}}, \mathbf{w}\right)
\end{aligned}
$$

with $x, y, z, f_{p}$ and $g_{p}$ as in (2) and (3), together with the estimation errors defined by

$$
\widetilde{x}_{p}:=x_{p}-x, \quad \widetilde{y}_{p}:=y_{p}-y, \quad \widetilde{z}_{p}:=z_{p}-z .
$$

For each $p \in \mathcal{P}$, let us denote $\left(x_{p}, y_{p}, z_{p}\right)^{T}$ by $\mathbf{x}_{p}$ and $\left(\widetilde{x}_{p}, \widetilde{y}_{p}, \widetilde{z}_{p}\right)^{T}$ by $\mathbf{e}_{p}$, to have $\mathbf{e}_{p}=\mathbf{x}_{p}-\mathbf{x}_{\mathrm{i}}$. It follows from (4) and (8) that $\dot{\mathbf{e}}_{p^{*}}=-\mathbf{e}_{p^{*}}$ for any control $\mathbf{w}$, thus

$$
\mathbf{e}_{p^{*}}(t)=e^{-t} \mathbf{e}_{p^{*}}(0), \quad t \geq 0 .
$$

\subsection{Multi-Controller}

For each $p \in \mathcal{P}$, when $x_{p}^{2}+y_{p}^{2} \neq 0$, the equations

$$
x_{p}=r_{p} \cos \psi_{p}, \quad y_{p}=r_{p} \sin \psi_{p}
$$

define the signals $r_{p}$ and $\psi_{p}$. There exists a function $h_{p}: \mathbb{R}^{9} \rightarrow \mathbb{R}^{2}$ such that for all $\mathbf{x}_{\mathrm{u}}, \mathbf{x}_{p}$ and $\mathbf{e}_{p}$ with $x_{p}^{2}+y_{p}^{2} \neq 0$ we have

$$
\begin{aligned}
& f_{p}\left(\mathbf{x}_{\mathrm{u}}, h_{p}\left(\mathbf{x}_{\mathrm{u}}, \mathbf{x}_{p}, \mathbf{e}_{p}\right)\right)=-x_{p} r_{p}+z_{p} \sin \psi_{p}+\widetilde{x}_{p} \\
& g_{p}\left(\mathbf{x}_{\mathrm{u}}, h_{p}\left(\mathbf{x}_{\mathrm{u}}, \mathbf{x}_{p}, \mathbf{e}_{p}\right)\right)=-y_{p} r_{p}-z_{p} \cos \psi_{p}+\widetilde{y}_{p}
\end{aligned}
$$

Similarly, there exists a function $\bar{h}_{p}: \mathbb{R}^{3} \rightarrow \mathbb{R}^{2}$ such that for all $\mathbf{x}_{\mathrm{u}}$ we have

$$
f_{p}\left(\mathbf{x}_{\mathrm{u}}, \bar{h}_{p}\left(\mathbf{x}_{\mathrm{u}}\right)\right)=1, \quad g_{p}\left(\mathbf{x}_{\mathrm{u}}, \bar{h}_{p}\left(\mathbf{x}_{\mathrm{u}}\right)\right)=1 .
$$

These functions are given explicitly by the formulas

$$
\begin{aligned}
h_{p}\left(\mathbf{x}_{\mathrm{u}}, \mathbf{x}_{p}, \mathbf{e}_{p}\right) & =\left(\begin{array}{c}
\left(\left(x_{1} \sin \theta-x_{2} \cos \theta\right) \Omega+\widetilde{x}_{p}-x_{p} r_{p}+z_{p} \sin \psi_{p}\right) / p_{1} \\
\Omega / p_{2}
\end{array}\right) \\
\bar{h}_{p}\left(\mathbf{x}_{\mathrm{u}}\right) & =\left(\begin{array}{c}
\left(1+x_{1} \sin \theta-x_{2} \cos \theta\right) / p_{1} \\
1 / p_{2}
\end{array}\right)
\end{aligned}
$$


where $\Omega=\widetilde{y}_{p}-y_{p} r_{p}-z_{p} \cos \psi_{p}$. Note that $\mathbf{x}_{\mathrm{u}}$ can be computed from $\mathbf{x}_{p}$ and $\mathbf{e}_{p}$ via (2) and (9), so that the first argument of the function $h_{p}$ is actually superfluous. This explains the fact that the only inputs to the multi-controller in Figure 2 are $\mathbf{x}_{p}$ and $\mathbf{e}_{p}$.

Our hybrid control law will depend on two discrete state variables generated by the switching logic to be described below: $\sigma \in \mathcal{P}$ (the current estimate of $p^{*}$ ) and $s \in\{0,1\}$ (an auxiliary logic variable). We define the candidate control laws $\boldsymbol{\rho}_{p}, p \in \mathcal{P}$ by

$$
\boldsymbol{\rho}_{p}\left(s, \mathbf{x}_{\mathrm{u}}, \mathbf{x}_{p}, \mathbf{e}_{p}\right):= \begin{cases}h_{p}\left(\mathbf{x}_{\mathrm{u}}, \mathbf{x}_{p}, \mathbf{e}_{p}\right) & \text { if } s=1 \text { and } r_{p} \neq 0 \\ \bar{h}_{p}\left(\mathbf{x}_{\mathrm{u}}\right) & \text { otherwise }\end{cases}
$$

Loosely speaking, each candidate control law $\boldsymbol{\rho}_{p}\left(s, \mathbf{x}_{\mathbf{u}}, \mathbf{x}_{p}, \mathbf{e}_{p}\right)$ makes the corresponding estimator state $\mathbf{x}_{p}$ reproduce the desired behavior given by (7) as closely as possible (see the equations (19) and (20) below). In fact, we would get precisely the equations (7) if $\mathbf{e}_{p}$ were equal to zero. Now the motivation for considering the above candidate control laws can be seen more clearly: we know that at least for $p=p^{*}$ the estimation error $\mathbf{e}_{p}$ converges to 0 . Thus, if we had $\sigma \rightarrow p^{*}$, then the control values would converge to those of the control law that would have been implemented in the case of known $p^{*}$. However, in supervisory control the parameter estimates are not required to converge to the actual parameter values, and typically will not do so. We will see that this is not crucial in proving stability of the closed-loop system.

\subsection{Performance signals}

Define a function $F: \mathbb{R}^{3} \rightarrow \mathbb{R}$ by

$$
F(v):=v_{1}^{2}+v_{2}^{2}+v_{2}^{6}+v_{3}^{2} .
$$

Fix a positive number $\lambda<2$. Each performance signal $\pi_{p}, p \in \mathcal{P}$ will be generated by

$$
\dot{\pi}_{p}=-\lambda \pi_{p}+F\left(\mathbf{e}_{p}\right)
$$

with the initial values satisfying $\pi_{p}(0)>0$. The equation (15) implies that for each $p \in \mathcal{P}$ we have

$$
\pi_{p}(t)=e^{-\lambda t} \pi_{p}(0)+\int_{0}^{t} e^{-\lambda(t-\tau)} F\left(\mathbf{e}_{p}(\tau)\right) d \tau, \quad t \geq 0 .
$$

This formula shows that each performance signal $\pi_{p}$ is the sum of an exponentially decaying term that depends on initial conditions and a suitable exponentially weighted "norm" of the corresponding estimation errors. The reason for defining the performance signals in this manner will become clear later.

\subsection{Switching logic}

The switching logic we consider here is a modified version of the scale-independent hysteresis switching logic proposed in $[7,8]$. Let us pick a number $h>0$ called the hysteresis constant. The functioning of the switching logic is as follows (see Figure 3). First, we set $\sigma(0)=\arg \min _{p \in \mathcal{P}}\left\{\pi_{p}(0)\right\}$. Suppose that at a certain time $t_{i}$ the value of $\sigma$ has just switched to some $q \in \mathcal{P}$. If $r_{q}\left(t_{i}\right) \neq 0$, we set $s=1$. The value of $\sigma$ is then held fixed until a time $t_{i+1}>t_{i}$ such that $(1+h) \min _{p \in \mathcal{P}}\left\{\pi_{p}\left(t_{i+1}\right)\right\} \leq \pi_{q}\left(t_{i+1}\right)$, at which point we set $\sigma\left(t_{i+1}\right)=\arg \min _{p \in \mathcal{P}}\left\{\pi_{p}\left(t_{i}+1\right)\right\}$. (When the indicated minimum is not unique, a particular value among those that minimize $\pi_{p}$ can be chosen arbitrarily.) On the other hand, if $r_{q}\left(t_{i}\right)=0$, we set $s=0$ and keep 
the value of $\sigma$ fixed for $t \in\left[t_{i}, t_{i}+\delta\right)$, where $\delta$ is a positive constant. After that we set $s=1$ and go back to the first case. Repeating this procedure, we generate a piecewise constant signal $\sigma$ that is continuous from the right everywhere. As we will see, by setting $\pi_{p}(0)>0$ for all $p \in \mathcal{P}$ we avoid chattering.

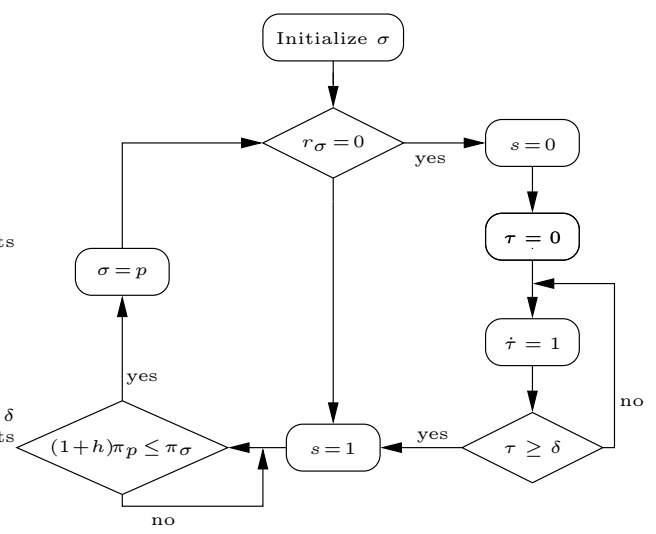

Figure 3: Switching logic

Finally, using the candidate control laws (14) and the above switching logic, we define the hybrid feedback control law by

$$
\mathbf{w}:=\rho_{\sigma}
$$

\subsection{Main result}

Our main result is as follows.

Theorem 1 All the signals in the supervisory control system defined by (1), (8), (14), (15), (16) and the switching logic of Section 3.4 remain bounded for any initial conditions such that $\pi_{p}(0)>0 \forall p \in \mathcal{P}$. Moreover, there exists a time $T^{*}$ such that $\sigma(t)=q^{*} \in \mathcal{P}$ for all $t \geq T^{*}$, i.e., the switching stops in finite time, and we have $x_{1}(t), x_{2}(t), \theta(t) \rightarrow 0$ as $t \rightarrow \infty$.

To prove this theorem, we will need the following well known and easily derived facts.

Fact 2 The state of an exponentially stable linear system with bounded inputs and/or $L^{k}$ inputs ( $k \geq 1$ ) remains bounded.

Fact 3 (see, e.g., [1, p. 58]) Any $L^{2}$ function with bounded derivative converges to zero.

Lemma 4 Consider the system

$$
\dot{x}=a(t) x+b(t) .
$$

If $T>0$ is such that $\int_{0}^{T} a(t) d t<\infty$ and $\int_{0}^{T}|b(t)| d t<\infty$, then $x(t)$ is bounded for $t \in[0, T)$. Moreover, if $\lim _{T \rightarrow \infty} \int_{0}^{T} a(t) d t=-\infty$ and $\lim _{T \rightarrow \infty} \int_{0}^{T}|b(t)| d t=N<\infty$, then $x(t) \rightarrow 0$ as $t \rightarrow \infty$.

Proof of Theorem 1. Let us define (for analysis purposes only) the scaled performance signals $\bar{\pi}_{p}(t):=$ $e^{\lambda t} \pi_{p}(t), p \in \mathcal{P}$. In view of (15) we have

$$
\dot{\bar{\pi}}_{p}=e^{\lambda t} F\left(\mathbf{e}_{p}\right)
$$


The scale independence property of the switching logic implies that replacing $\pi_{p}$ by $\bar{\pi}_{p}$ would have no effect on $\sigma$. From (18) we see that each $\bar{\pi}_{p}$ is monotonically nondecreasing. This, the finiteness of $\mathcal{P}$, and the fact that $\bar{\pi}_{p}(0)>0$ for each $p \in \mathcal{P}$ guarantee the existence of a positive number $\epsilon$ such that $\bar{\pi}_{p}(t)>\epsilon \forall t \geq 0, \forall p \in \mathcal{P}$. It is not hard to conclude now from the way the switching logic was defined that chattering cannot occur $[7,8]$.

Observe that $\bar{\pi}_{p^{*}}$ is bounded by virtue of (10). It follows that the signals $\bar{\pi}_{p}$ satisfy the assumptions of the Hysteresis Switching Lemma [7, 8, 15] which enables us to conclude that the switching stops in finite time $^{1}$. More precisely, if $[0, T)$ is the largest interval on which the solution to the system is defined, there exists a time $T^{*}<T$ such that $\sigma(t)=q^{*} \in \mathcal{P}$ for all $t \geq T^{*}$. In addition, $\bar{\pi}_{q^{*}}$ is bounded on $[0, T)$.

If $s\left(T^{*}\right)=0$, the composite state of the system remains bounded for all $t \in\left[T^{*}, T^{*}+\delta\right]$ because the closed-loop system is globally Lipschitz. We will then have $s\left(T^{*}+\delta\right)=1$. From (8), (12), (14) and (16), using straightforward calculations, we conclude that for $t \geq T^{*}+\delta$ we have

$$
\begin{aligned}
& \dot{r}_{q^{*}}=-r_{q^{*}}^{2} \\
& \dot{z}_{q^{*}}=-z_{q^{*}}\left(r_{q^{*}}-\widetilde{x}_{q^{*}} \cos \psi_{q^{*}}-\widetilde{y}_{q^{*}} \sin \psi_{q^{*}}\right)+\left(x_{q^{*}} \widetilde{y}_{q^{*}}-\widetilde{x}_{q^{*}} y_{q^{*}}\right)\left(1-r_{q^{*}}\right)-\widetilde{z}_{q^{*}}
\end{aligned}
$$

(here and below we are using the notation $r_{p}=\sqrt{x_{p}^{2}+y_{p}^{2}}, p \in \mathcal{P}$ ). The equation (19) and the definition of the switching logic imply that $r_{q^{*}}(t) \neq 0$ and $s(t)=1$ for all $t \geq T^{*}+\delta$. Thus we may assume, without loss of generality, that $s\left(T^{*}\right)=1$ and that (19)-(20) hold for $t \geq T^{*}$. For each $p \in \mathcal{P} \backslash q^{*}$ and all $t \geq T^{*}$ from (8), (12), (14) and (16) we obtain the equations

$$
\begin{aligned}
& \dot{r}_{p}=-r_{p}+\xi_{p q^{*}}\left(\mathbf{x}_{q^{*}}, \mathbf{e}_{q^{*}}\right) \\
& \dot{z}_{p}=-z_{p}+\eta_{p q^{*}}\left(\mathbf{x}_{q^{*}}, \mathbf{e}_{q^{*}}\right)
\end{aligned}
$$

Here $\xi_{p q^{*}}$ and $\eta_{p q^{*}}$ are appropriately defined functions which satisfy the bounds

$$
\begin{aligned}
& \xi_{p q^{*}}\left(\mathbf{x}_{q^{*}}, \mathbf{e}_{q^{*}}\right) \leq P_{1}\left(r_{q^{*}}, \mathbf{x}_{q^{*}}\right) P_{2}\left(\widetilde{x}_{q^{*}}\right)+P_{3}\left(r_{q^{*}}, \mathbf{x}_{q^{*}}\right) P_{4}\left(\widetilde{y}_{q^{*}}\right)+P_{5}\left(r_{q^{*}}, \mathbf{x}_{q^{*}}\right) P_{6}\left(\widetilde{z}_{q^{*}}\right) \\
& \eta_{p q^{*}}\left(\mathbf{x}_{q^{*}}, \mathbf{e}_{q^{*}}\right) \leq P_{7}\left(r_{q^{*}}, \mathbf{x}_{q^{*}}\right) P_{8}\left(\widetilde{x}_{q^{*}}\right)+P_{9}\left(r_{q^{*}}, \mathbf{x}_{q^{*}}\right) P_{10}\left(\widetilde{y}_{q^{*}}\right)+P_{11}\left(r_{q^{*}}, \mathbf{x}_{q^{*}}\right) P_{12}\left(\widetilde{z}_{q^{*}}\right)
\end{aligned}
$$

where $P_{i}$ 's are suitably defined polynomials. In particular, $\operatorname{deg} P_{2}=\operatorname{deg} P_{6}=\operatorname{deg} P_{8}=\operatorname{deg} P_{12}=2$, $\operatorname{deg} P_{4}=4$, and $\operatorname{deg} P_{10}=6$.

It follows from (19) that

$$
r_{q^{*}}(t)=\frac{r_{q^{*}}(0)}{r_{q^{*}}(0) t+1}, \quad t \in[0, T) .
$$

Since $\bar{\pi}_{q^{*}}$ is bounded, we have $e^{\lambda t} F\left(\mathbf{e}_{q^{*}}\right) \in L^{1}([0, T))$. This implies, in particular, that $\widetilde{x}_{q^{*}}, \widetilde{z}_{q^{*}} \in L^{2}([0, T))$ and $\widetilde{y}_{q^{*}} \in L^{2}([0, T)) \cap L^{6}([0, T))$. Also, $\widetilde{y}_{q^{*}} \in L^{4}([0, T))$ by virtue of the bound $\widetilde{y}_{q^{*}}^{4} \leq\left(\widetilde{y}_{q^{*}}^{2}+\widetilde{y}_{q^{*}}^{6}\right) / 2$. Moreover, from the bound $\left|\widetilde{x}_{q^{*}}\right| \leq\left(e^{-\lambda t}+e^{\lambda t} \widetilde{x}_{q^{*}}^{2}\right) / 2$ we see that $\widetilde{x}_{q^{*}} \in L^{1}([0, T))$. Similarly, $\widetilde{y}_{q^{*}}, \widetilde{z}_{q^{*}} \in L^{1}([0, T))$. One can easily check now that the equation (20) satisfies the assumptions of Lemma 4 . Combined with the polynomial bounds (22) and (23) this implies that $\mathbf{x}_{p}$ is bounded on $[0, T)$ for all $p \in \mathcal{P}$ (here we are using (21) and Fact 2). In view of (9) and (10) we conclude that $\mathbf{x}_{\mathrm{i}}$ remains bounded as well. Therefore, $\mathbf{e}_{p}$ is bounded for each $p \in \mathcal{P}$. The boundedness of all the performance signals now follows from Fact 2 applied to (15). Thus we see that $T=\infty$, i.e., the solution to the system is globally defined.

\footnotetext{
${ }^{1}$ Although the presence of the variable $s$ makes the switching logic described here more complicated than the ones analyzed in the cited references, this does not introduce any difficulties in establishing the desired finite number of switchings property.
} 
Now (19)-(20) and Lemma 4 imply that $\mathbf{x}_{q^{*}} \rightarrow 0$. Furthermore, since $\mathbf{x}_{\mathbf{i}}$ and $\mathbf{x}_{p}, p \in \mathcal{P}$ are bounded, $\dot{\widetilde{x}}_{q^{*}}=\dot{x}_{q^{*}}-\dot{x}$ is bounded as well. We also know that $\widetilde{x}_{q^{*}} \in L^{2}$. Using Fact 3 , we conclude that $\widetilde{x}_{q^{*}} \rightarrow 0$. Similarly, $\widetilde{y}_{q^{*}}, \widetilde{z}_{q^{*}} \rightarrow 0$. Therefore, we have $\mathbf{x}_{\mathrm{i}} \rightarrow 0$, and consequently $\mathbf{x}_{\mathrm{u}} \rightarrow 0$ as needed.

REMARK. The main result of [9] states, loosely speaking, that if for each $p \in \mathcal{P}$ the $p$-th controller input-tostate stabilizes the multi-estimator, with the estimation error $e_{p}$ viewed as the input, then the closed-loop system is detectable through $e_{p}$ when the value of $\sigma$ is frozen at $p$. That paper shows how this result leads to a systematic supervisory control design technique with the help of suitable supply functions (for example, in the linear case one can always choose these functions to be quadratic, hence the performance signals can be driven by the squared estimation errors - cf. Appendix). However, even when the input-to-state stabilization hypothesis is not satisfied, it is sometimes possible to establish the desired properties of the closed-loop system directly along the lines of the above proof. Integral variants of input-to-state stability recently introduced in [19] are also relevant in this regard, as discussed in [11].

\section{Practical regulation}

As we remarked earlier, the solutions of (7) do not converge to zero exponentially. We therefore expect that the control strategy developed in the previous section will cause slow convergence. In some applications it might be sufficient to make the state $\mathbf{x}_{\mathrm{u}}$ of the system (1) approach a small region around the origin and stay there for all future time (cf. [6]). In view of the developments of Section 2, this task will be accomplished if we drive the state of the nonholonomic integrator (4) to a sufficiently small neighborhood of the origin. In this section we describe how this problem can be solved.

Consider the problem of controlling the state of the system (5) so as to have $r \rightarrow r^{*}, z \rightarrow 0$, where $r^{*}$ is a (small) fixed positive real number. The control law

$$
\bar{u}_{p^{*}}=-r+r^{*}, \quad \bar{v}_{p^{*}}=-\frac{z}{r^{*}}
$$

proposed in [5] yields the exponentially stable system

$$
\begin{aligned}
& \dot{\bar{r}}=-\bar{r} \\
& \dot{z}=-\frac{z r}{r^{*}}
\end{aligned}
$$

where $\bar{r}:=r-r^{*}$.

Since we are dealing with the case when the actual parameter values are unknown, the above control law cannot be implemented. Let us consider again the estimator equations given by (8) together with the estimation errors given by (9). We will design the candidate control laws in such a way as to make the estimators reproduce the desired behavior given by (24) as closely as possible (see the equations (28) below). The motivation for considering such candidate control laws is the same as before.

For each $p \in \mathcal{P}$ there exists a function $k_{p}: \mathbb{R}^{9} \rightarrow \mathbb{R}^{2}$ such that for all $\mathbf{x}_{\mathrm{u}}, \mathbf{x}_{p}$ and $\mathbf{e}_{p}$ with $x_{p}^{2}+y_{p}^{2} \neq 0$ we have

$$
\begin{aligned}
& f_{p}\left(\mathbf{x}_{\mathrm{u}}, k_{p}\left(\mathbf{x}_{\mathrm{u}}, \mathbf{x}_{p}, \mathbf{e}_{p}\right)\right)=-x_{p}+r^{*} \cos \psi_{p}+\frac{z_{p} \sin \psi_{p}}{r^{*}}+\widetilde{x}_{p} \\
& g_{p}\left(\mathbf{x}_{\mathrm{u}}, k_{p}\left(\mathbf{x}_{\mathrm{u}}, \mathbf{x}_{p}, \mathbf{e}_{p}\right)\right)=-y_{p}+r^{*} \sin \psi_{p}-\frac{z_{p} \cos \psi_{p}}{r^{*}}+\widetilde{y}_{p}
\end{aligned}
$$


where $\psi_{p}$ is defined by (11). This function is given explicitly by the formula

$$
k_{p}\left(\mathbf{x}_{\mathrm{u}}, \mathbf{x}_{p}, \mathbf{e}_{p}\right)=\left(\begin{array}{c}
\left(\left(x_{1} \sin \theta-x_{2} \cos \theta\right) \Upsilon-x_{p}+r^{*} \cos \psi_{p}+\frac{z_{p} \sin \psi_{p}}{r^{*}}+\widetilde{x}_{p}\right) / p_{1} \\
\Upsilon / p_{2}
\end{array}\right)
$$

where $\Upsilon=-y_{p}+r^{*} \sin \psi_{p}-\frac{z_{p} \cos \psi_{p}}{r^{*}}+\widetilde{y}_{p}$. As before, the first argument of $k_{p}$ is actually superfluous.

Our hybrid control law will depend on two discrete state variables generated by the switching logic described in Section 3.4: $\sigma \in \mathcal{P}$ and $s \in\{0,1\}$. We define the candidate control laws $\overline{\boldsymbol{\rho}}_{p}, p \in \mathcal{P}$ by

$$
\overline{\boldsymbol{\rho}}_{p}\left(s, \mathbf{x}_{\mathrm{u}}, \mathbf{x}_{p}, \mathbf{e}_{p}\right):= \begin{cases}k_{p}\left(\mathbf{x}_{\mathrm{u}}, \mathbf{x}_{p}, \mathbf{e}_{p}\right) & \text { if } s=1 \text { and } r_{p} \neq 0 \\ \bar{h}_{p}\left(\mathbf{x}_{\mathrm{u}}\right) & \text { otherwise }\end{cases}
$$

the function $\bar{h}_{p}$ having been defined by (13). Using these candidate control laws and the switching logic described in Section 3.4, we define the hybrid feedback control law by

$$
\mathbf{w}:=\overline{\boldsymbol{\rho}}_{\sigma} .
$$

This leads us to the following result.

Theorem 5 All the signals in the supervisory control system defined by (1), (8), (15), (26), (27) and the switching logic of Section 3.4 remain bounded for any initial conditions such that $\pi_{p}(0)>0 \forall p \in \mathcal{P}$. Moreover, there exists a time $T^{*}$ such that $\sigma(t)=q^{*} \in \mathcal{P}$ for all $t \geq T^{*}$, i.e., the switching stops in finite time, and we have $r(t) \rightarrow r^{*}$ and $z(t) \rightarrow 0$ as $t \rightarrow \infty$.

Proof. As before, we can apply an appropriate version of the Hysteresis Switching Lemma to show that if $(0, T)$ is the largest interval on which the solution to the system is defined, there exists a time $T^{*}<T$ such that $\sigma(t)=q^{*} \in \mathcal{P}$ for all $t \geq T^{*}$. We can also assume, without loss of generality, that $s\left(T^{*}\right)=1$. From (8), (25), (26) and (27), using straightforward calculations, we obtain the equations

$$
\begin{aligned}
& \dot{r}_{q^{*}}=-r_{q^{*}}+r^{*} \\
& \dot{z}_{q^{*}}=-\frac{z_{q^{*}}}{r^{*}}\left(r_{q^{*}}-\widetilde{x}_{q^{*}} \cos \psi_{q^{*}}-\widetilde{y}_{q^{*}} \sin \psi_{q^{*}}\right)+r^{*}\left(\widetilde{y}_{q^{*}} \cos \psi_{q^{*}}-\widetilde{x}_{q^{*}} \sin \psi_{q^{*}}\right)-\widetilde{z}_{q^{*}}
\end{aligned}
$$

and, for each $p \in \mathcal{P} \backslash q^{*}$,

$$
\begin{aligned}
& \dot{r}_{p}=-r_{p}+\bar{\xi}_{p q^{*}}\left(\mathbf{x}_{q^{*}}, \mathbf{e}_{q^{*}}\right) \\
& \dot{z}_{p}=-z_{p}+\bar{\eta}_{p q^{*}}\left(\mathbf{x}_{q^{*}}, \mathbf{e}_{q^{*}}\right)
\end{aligned}
$$

Here $\bar{\xi}_{p q^{*}}$ and $\bar{\eta}_{p q^{*}}$ are appropriately defined functions which have exactly the same properties as the functions $\xi_{p q^{*}}$ and $\eta_{p q^{*}}$ introduced in the proof of Theorem 1. The rest of the proof carries over from Theorem 1 with obvious slight modifications and will not be given.

\section{State-sharing}

In the previous section we have relied on the fact that the set $\mathcal{P}$ was finite, so that the systems of estimator equations (8) and performance signal equations (15) were finite-dimensional. If the set $\mathcal{P}$ is infinite, a different approach is required. One alternative is to replace the individual estimator equations by a single 
system and use it to generate the estimation errors, in other words, make the estimators "share" the same state. The performance signals can be obtained in a similar way. This idea in fact leads to a more efficient way of designing a supervisory control system even for the case when $\mathcal{P}$ has a finite but large number of elements. What makes such state sharing possible in our case is the fact that the control functions $f_{p}\left(\mathbf{x}_{\mathrm{u}}, \mathbf{w}\right)$ and $g_{p}\left(\mathbf{x}_{\mathrm{u}}, \mathbf{w}\right)$ are separable, i.e., take the form $a^{T}(p) b\left(\mathbf{x}_{\mathrm{u}}, \mathbf{w}\right)$.

Consider the equations

$$
\begin{array}{lll}
\dot{\hat{x}}_{1}=-\widehat{x}_{1}+x & \dot{\hat{\widehat{y}}}_{1}=-\widehat{\hat{z}}_{1}+z \\
\dot{\hat{x}}_{2}=-\widehat{x}_{2}+w_{1} & \dot{\hat{y}}_{2}=-\widehat{\hat{y}}_{2}+w_{2}=-\widehat{z}_{2}-y w_{1} \\
\dot{\hat{x}}_{3}=-\widehat{x}_{3}-w_{2} \frac{x y+z}{2} & \dot{\hat{z}}_{3}=-\widehat{z}_{3}+x w_{2}+y w_{2} \frac{x y+z}{2}
\end{array}
$$

with $x, y$ and $z$ given by (2). If we now define

$$
x_{p}:=\widehat{x}_{1}+p_{1} \widehat{x}_{2}+p_{2} \widehat{x}_{3}, \quad y_{p}:=\widehat{y}_{1}+p_{2} \widehat{y}_{2}, \quad z_{p}:=\widehat{z}_{1}+p_{1} \widehat{z}_{2}+p_{2} \widehat{z}_{3}
$$

then it is easy to check that $x_{p}, y_{p}$ and $z_{p}$ satisfy the original estimator equations (8). We thus define the $p$-th estimation error $\mathbf{e}_{p}=\left(\widetilde{x}_{p}, \widetilde{y}_{p}, \widetilde{z}_{p}\right)^{T}$ by

$$
\widetilde{x}_{p}:=\widehat{x}_{1}+p_{1} \widehat{x}_{2}+p_{2} \widehat{x}_{3}-x, \quad \widetilde{y}_{p}:=\widehat{y}_{1}+p_{2} \widehat{y}_{2}-y, \quad \widetilde{z}_{p}:=\widehat{z}_{1}+p_{1} \widehat{z}_{2}+p_{2} \widehat{z}_{3}-z
$$

and use (30)-(31) in place of (8).

The performance signals can be obtained similarly. As for the candidate control laws and the switching logic, they can be defined exactly as before. One can check that the minimization procedure used in the switching algorithm reduces to that of finding roots of polynomials in one variable of degree at most 5 , so its computational tractability is not an issue.

The above discussion explains how to implement the supervisory control algorithms in the case when the parameter set $\mathcal{P}$ is infinite. Once this is done, the question that arises is how to prove that these algorithms indeed perform the required tasks. Unfortunately, the switching cannot be guaranteed to stop in this case, and the problem of proving the state convergence remains open.

However, simulation results indicate that the closed-loop system still has the desired convergence property, as is illustrated by the figures below. We took the set $\mathcal{P}$ to be $[0.1,2] \times[0.1,3]$ and the actual parameter pair to be $p^{*}=(1,1)$. Figure 4 shows the behavior of the state $\mathbf{x}_{\mathrm{u}}$ when one applies the control law of Section 3 and the control law of Section 4 (with $r^{*}=0.1$ ), respectively. On the basis of this and other simulation studies we have concluded that the state of the system is indeed driven to zero in the first case and to an appropriate neighborhood of zero in the second case. These simulations also suggest that, loosely speaking, the second control law tends to bring the state of the system into a "reasonably small" neighborhood of zero faster than the first one, which is not surprising in view of our earlier discussion ${ }^{2}$.

\section{Conclusions}

In this paper we used the supervisory control approach to solve a prototype control problem for a nonholonomic system with parametric modeling uncertainty. The results of [17] lead us to believe that a fairly

\footnotetext{
${ }^{2}$ A parking movie generated with MATLAB Simulink which illustrates the performance of the supervisory control system designed in Section 3 is available at http://pantheon.yale.edu/ ${ }^{\mathrm{dml} l 33 / \text { parkingmovie.mpg }}$
} 

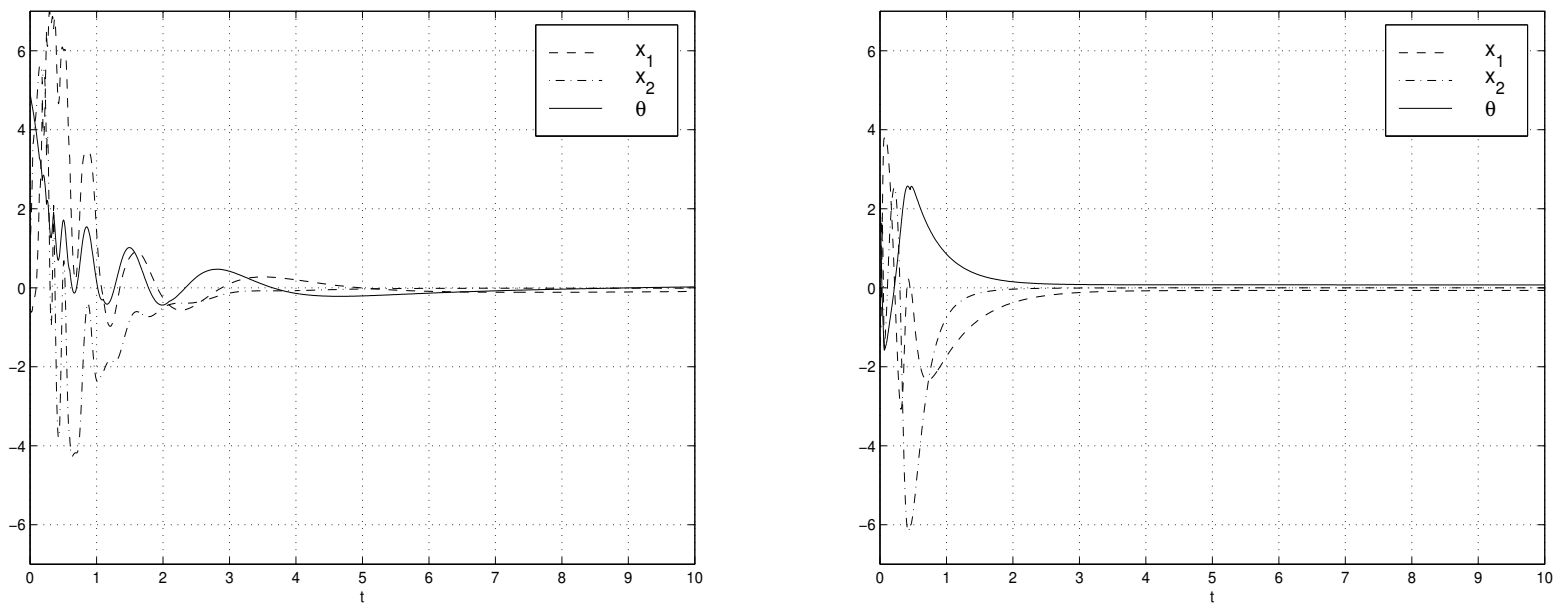

Figure 4: Exact and practical regulation

general class of nonholonomic systems can be treated by the method proposed in this paper. It would be interesting to compare our approach with existing adaptive control algorithms, e.g., the one presented by Jiang and Pomet in [12].

The main open problem that remains the subject of ongoing research efforts is to prove that the algorithms described here still perform the required tasks, or to develop other provably correct algorithms, when the switching cannot be guaranteed to stop. This issue arises when the parameter set is infinite or, perhaps more importantly, when disturbances, noise, and/or unmodeled dynamics are present.

\section{Appendix}

In this appendix we discuss, in the context of a simple example, some aspects of the supervisory control machinery developed in [16] that are relevant to the work described in this paper. Consider the problem of stabilizing, by means of output feedback, an unknown member of a family of stabilizable and detectable linear systems

$$
\dot{x}=A_{p} x+B_{p} u ; \quad y=C_{p} x
$$

where $x \in \mathbb{R}^{n}, u \in \mathbb{R}^{m}, y \in \mathbb{R}^{k}$, and the parameter $p$ takes values in a finite index set $\mathcal{P}$. We will write $p^{*}$ for the actual value of the parameter that corresponds to the system to be stabilized. If the value of $p^{*}$ were known, one could apply, for example, the standard dynamic observer-based linear feedback control law to stabilize the system. Since the actual parameter value is not available, we would like to design a family of candidate control laws and orchestrate the switching between them based on some parameter estimation procedure. To this end, consider a family of observer-based output estimators parameterized by $\mathcal{P}$ which take the form

$$
\dot{x}_{p}=\left(A_{p}+K_{p} C_{p}\right) x_{p}+B_{p} u-K_{p} y ; \quad y_{p}=C_{p} x_{p}
$$

and the corresponding candidate control laws

$$
u_{p}=F_{p} x_{p}
$$


Here the matrices $K_{p}$ and $F_{p}$ are such that the eigenvalues of $A_{p}+K_{p} C_{p}$ and $A_{p}+B_{p} F_{p}$ have negative real parts for each $p \in \mathcal{P}$ (such matrices exist because each system in the family (32) is stabilizable and detectable by assumption).

We also consider the estimation, or output prediction, errors defined for each $p \in \mathcal{P}$ by

$$
e_{p}:=y_{p}-y
$$

and the corresponding performance signals defined for each $p \in \mathcal{P}$ by

$$
\pi_{p}(t):=\int_{0}^{t}\left\|e_{p}(\tau)\right\|^{2} d \tau .
$$

Each $\pi_{p}(t)$ can be thought of as the $L^{2}$ norm of $e_{p}$ on the interval $[0, t]$. It contains information about the past behavior of the estimation error $e_{p}$ and can be regarded as a measure of the likelihood of $p$ being the actual parameter value $p^{*}$. Note that each performance signal can be generated by the differential equation

$$
\dot{\pi}_{p}=\left\|e_{p}\right\|^{2}
$$

with $\pi_{p}(0)=0$.

We will design a hybrid feedback control policy of the form $u=u_{\sigma}$, where $\sigma:[0, \infty) \rightarrow \mathcal{P}$ is a piecewise constant switching signal. In our case this means $u(t)=F_{\sigma(t)} x_{\sigma(t)}$. Loosely speaking, the value of $\sigma$ at each given time will correspond to the parameter $p$ whose associated performance index $\pi_{p}$ is currently the smallest. The origin of this idea is the concept known as certainty equivalence in the adaptive context or as separation principle in the stochastic context. The justification of this approach comes from the fact that, as we will show below, the closed-loop system is detectable through the estimation error.

One way to generate the switching signal $\sigma$ is by means of the so-called hysteresis switching logic [15]. Fix a positive number $h$ called the hysteresis constant. Set $\sigma(0)=\arg \min _{p \in \mathcal{P}}\left\{\pi_{p}(0)\right\}$. Now, suppose that at a certain time $\sigma$ has just switched to some $q \in \mathcal{P}$. The value of $\sigma$ is then held fixed until we have $\min _{p \in \mathcal{P}}\left\{\pi_{p}(t)\right\}+h \leq \pi_{q}(t)$. If and when that happens, we set $\sigma$ equal to $\arg \min _{p \in \mathcal{P}}\left\{\pi_{p}(t)\right\}$. Repeating this procedure, we obtain a piecewise constant switching signal which is continuous from the right everywhere.

The equations (32), (33) and (35) imply that for any control law $u$ we have $\dot{e}_{p^{*}}=-e_{p^{*}}$, so that $e_{p^{*}}(t)=$ $e^{-t} e_{p^{*}}(0)$. It follows at once from (36) that $\pi_{p^{*}}(t)$ is bounded for all $t \geq 0$. In addition, all performance signals $\pi_{p}$ are nondecreasing by construction. Using these two facts and the definition of the hysteresis switching logic, it is not hard to show that the switching must stop in finite time (this is the Hysteresis Switching Lemma proved in [15]). More precisely, there exists a time $T^{*}$ such that $\sigma(t)=q^{*} \in \mathcal{P} \forall t \geq T^{*}$. Moreover, $\pi_{q^{*}}$ is bounded because $\pi_{p^{*}}$ is, hence

$$
e_{q^{*}} \in L^{2}
$$

by virtue of (36).

After the switching stops, the closed-loop system can be written as

$$
\left(\begin{array}{c}
\dot{x} \\
\dot{x}_{q^{*}}
\end{array}\right)=\bar{A}\left(\begin{array}{c}
x \\
x_{q^{*}}
\end{array}\right) ; \quad e_{q^{*}}=\bar{C}\left(\begin{array}{c}
x \\
x_{q^{*}}
\end{array}\right)
$$

where

$$
\bar{A}:=\left(\begin{array}{cc}
A_{p^{*}} & B_{p^{*}} F_{q^{*}} \\
-K_{q^{*}} C_{p^{*}} & A_{q^{*}}+K_{q^{*}} C_{q^{*}}+B_{q^{*}} F_{q^{*}}
\end{array}\right)
$$


and

$$
\bar{C}:=\left(\begin{array}{ll}
-C_{p^{*}} & C_{q^{*}}
\end{array}\right)
$$

If we let

$$
\bar{K}:=\left(\begin{array}{l}
K_{p^{*}} \\
K_{q^{*}}
\end{array}\right)
$$

then it is straightforward to check that the matrix

$$
\bar{A}-\bar{K} \bar{C}=\left(\begin{array}{cc}
A_{p^{*}}+K_{p^{*}} C_{p^{*}} & B_{p^{*}} F_{q^{*}}-K_{p^{*}} C_{q^{*}} \\
0 & A_{q^{*}}+B_{q^{*}} F_{q^{*}}
\end{array}\right) .
$$

is strictly stable, which shows that the system (38) is detectable through $e_{q^{*}}$. To prove that $x$ converges to zero, it suffices to apply a standard output injection argument. Namely, write

$$
\left(\begin{array}{c}
\dot{x} \\
\dot{x}_{q^{*}}
\end{array}\right)=(\bar{A}-\bar{K} \bar{C})\left(\begin{array}{c}
x \\
x_{q^{*}}
\end{array}\right)+\bar{K} e_{q^{*}}
$$

and observe that $x \rightarrow 0$ in view of (37) and stability of $\bar{A}-\bar{K} \bar{C}$.

We emphasize that the particular choice of candidate control laws given by (34) is by no means crucial. Assume, for example, that every system in the family (32) is stabilizable by a static linear output feedback. In other words, assume that for each $p \in \mathcal{P}$ there exists a matrix $G_{p}$ such that the eigenvalues of $A_{p}+B_{p} G_{p} C_{p}$ have negative real parts. The reader will have no difficulty proving that if we keep the estimators (33) but replace (34) by $u_{p}=G_{p} y$, we still have $x \rightarrow 0$.

The above problem is rather special, and the solution and the method of proof have several drawbacks that we do not discuss here. Nonetheless, the essential features of this example are present in the supervisory control strategies developed in Sections 3 and 4.

\section{References}

[1] M. A. Aizerman, F. R. Gantmacher, Absolute Stability of Regulator Systems, Holden-Day, San Francisco, 1964.

[2] R. W. Brockett, Asymptotic stability and feedback stabilization, in Differential Geometric Control Theory (R. W. Brockett et al, Eds.), Birkhauser, Boston, 1983, pp. 181-191.

[3] R. Colbaugh, E. Barany, K. Glass, Adaptive control of nonholonomic mechanical systems, in Proc. 35th Conf. on Decision and Control, 1996, pp. 1428-1434.

[4] R. Colbaugh, Adaptive stabilization of mobile manipulators, in Proc. 1998 American Control Conf., pp. 1-5.

[5] G. Escobar, R. Ortega, M. Reyhanoglu, Regulation and tracking of the nonholonomic integrator: A field-oriented control approach, Automatica, vol. 34, 1998, pp. 125-131.

[6] R. Fierro, F. L. Lewis, Practical point stabilization of a nonholonomic mobile robot using neural networks, in Proc. 35th Conf. on Decision and Control, 1996, pp. 1722-1727.

[7] J. P. Hespanha, Logic-based switching algorithms in control, Ph.D. Thesis, Dept. of Electrical Engineering, Yale University, 1998.

[8] J. P. Hespanha, A. S. Morse, Scale-independent hysteresis switching, Technical report, Yale University, June 1998.

[9] J. P. Hespanha, A. S. Morse, Certainty equivalence implies detectability, Systems Control Lett., vol. 36, 1999, pp. 1-13.

[10] J. P. Hespanha, A. S. Morse, Stabilization of nonholonomic integrators via logic-based switching, Automatica (special issue on hybrid systems), vol. 35, 1999, pp. 385-393.

[11] J. P. Hespanha, A. S. Morse, Supervision of integral-input-to-state stabilizing controllers, in Proc. 5th European Control Conf., 1999, to appear. 
[12] Z. P. Jiang, J.-B. Pomet, Combining backstepping and time-varying techniques for a new set of adaptive controllers, in Proc. 33rd Conf. on Decision and Control, 1994, pp. 2207-2212.

[13] Z. P. Jiang, J.-B. Pomet, Global stabilization of parametric chained-form systems by time-varying dynamic feedback, Int. J. of Adaptive Control and Signal Processing, vol. 10, 1996, pp. 47-59.

[14] I. Kolmanovsky, N. H. McClamroch, Developments in nonholonomic control problems, IEEE Control Systems Magazine, vol. 15, 1995, pp. 20-36.

[15] A. S. Morse, D. Q. Mayne, G. C. Goodwin, Applications of hysteresis switching in parameter adaptive control, IEEE Trans. Automat. Control, vol. 37, 1992, pp. 1343-1354.

[16] A. S. Morse, Supervisory control of families of linear set-point controllers - part 1: Exact matching, IEEE Trans. Automat. Control, vol. 41, 1996, pp. 1413-1431.

[17] R. M. Murray, S. S. Sastry, Nonholonomic motion planning: Steering using sinusoids, IEEE Trans. Automat. Control, vol. 38, 1993, pp. 700-716.

[18] F. M. Pait, B. Piccoli, A hybrid controller for a nonholonomic system, in Proc. 30th Conf. on Inform. Sci. and Syst., 1996, pp. 416-420.

[19] E. D. Sontag, Comments on integral variants of ISS, Systems Control Lett., vol. 34, 1998, pp. 93-100.

[20] Y. Stepanenko, C.-Y. Su, Adaptive motion/force control of mechanical systems with nonholonomic Pfaffian constraints, in Proc. 1995 American Control Conf., pp. 375-379.

[21] V. Y. Tertychnyj, Integral estimation and adaptive stabilization of the controllable nonholonomic systems, J. Appl. Math. Mech, vol. 56, 1992, pp. 881-888. 